\title{
Human Gait Identification Using Persistent Homology
}

\author{
Javier Lamar-León, Edel B. García-Reyes, and Rocío Gonzalez-Diaz \\ Patterns Recognition Dept., Advanced Technologies Application Center, Cuba \\ Applied Math Dept., School of Computer Engineering, Univ. of Seville, Spain \\ $\{j$ lamar, egarcia\}@cenatav.co.cu, rogodi@us.es
}

\begin{abstract}
This paper shows an image/video application using topological invariants for human gait recognition. Using a background subtraction approach, a stack of silhouettes is extracted from a subsequence and glued through their gravity centers, forming a 3D digital image $I$. From this $3 \mathrm{D}$ representation, the border simplicial complex $\partial K(I)$ is obtained. We order the triangles of $\partial K(I)$ obtaining a sequence of subcomplexes of $\partial K(I)$. The corresponding filtration $F$ captures relations among the parts of the human body when walking. Finally, a topological gait signature is extracted from the persistence barcode according to $F$. In this work we obtain $98.5 \%$ correct classification rates on CASIA-B database 1 .
\end{abstract}

\section{Introduction}

Gait recognition is a challenging problem that gives the possibility to identify persons at a distance, without any interaction with the subjects, which is very important in real surveillance scenario 9 . Methods based on feature extraction using silhouettes or contour of silhouettes have been frequently used 3106]1. However, many silhouettes obtained during the process are incomplete due to illumination changes, occlusions, and others. These factors severely affect the recognition accuracy. Even though, a recent study $[3]$ is aimed at suppressing the effect of silhouette incompleteness to improve performance on previous approach, we do not pre-process the silhouettes in this paper.

The stability of geometric descriptors is affected by deformation in the silhouette shape. Even for the same individual, little changes on the walking direction, illumination variation and the way the clothes fit to the human body may cause variability of the geometric features. In this paper, the changes of silhouettes induced by gait are considered as a moving nonrigid object. In pattern recognition tasks, it is important to achieve an object description which is discriminative and invariant to different geometric transformations. Topological descriptions based on the persistence of the homology classes seem to be more invariant to these kind of transformations than classical approaches for gait recognition which are affected by changes and noise in the silhouette shape, as we have experimentally shown at the end of this paper. Even without improving the silhouettes, our

1 http://www.cbsr.ia.ac.cn/english/GaitDatabases.asp

L. Alvarez et al. (Eds.): CIARP 2012, LNCS 7441, pp. 244-251 2012.

(C) Springer-Verlag Berlin Heidelberg 2012 
results are better to the existing methods, like [3]. To the best of the authors knowledge this approach has never been applied to gait recognition.

Topology has been previously used to match nonrigid shapes 8 . Homology is a topological invariant frequently used in practice [2]. It could be a robust representation since the shapes of connected components and holes may change under geometric transformations, but the number of components and holes could remain the same. Nevertheless, it is not enough to reach the invariance for the representation, a set of discriminative features is also needed.

In this paper, a $2 \mathrm{D}$ border simplicial complex $\partial K(I)$ is obtained from a stack of silhouettes extracted from a subsequence and glued through their gravity centers. We then use incremental algorithm [5] to compute persistent homology on a particle filtration of $\partial K(I)$. We propose a novel topological representation for gait recognition from a simplification of the persistence barcode obtained from the given filtration. We test this representation on the CASIA-B database.

The rest of the paper is organized as follows. In Section 2 we explain how to obtain the simplicial complex $\partial K(I)$. We present a brief reviewing of the incremental algorithm for persistent homology in Section 3 . Section 4 is devoted to describe the new method in detail. Experimental results are then reported in Section 5. We conclude this paper and discuss some future work in Section 6 .

\section{The Simplicial Complex $\partial K(I)$}

First, the moving object (person) is segmented for each frame applying background modeling and subtraction. The sequence of silhouettes is analyzed to extract one subsequence of representation, which include at least a gait cycle [9]. One subsequence of representation is selected for each sequence.

The $3 \mathrm{D}$ binary digital picture $I=\left(\mathbb{Z}^{3}, 26,6, B\right)$ (where $B \subset \mathbb{Z}^{3}$ is the foreground, $B^{c}=\mathbb{Z}^{3} \backslash B$ the background, and $(26,6)$ is the adjacency relation for the foreground and background, respectively of a subsequence of representation is built stacking silhouettes aligned by their gravity centers $(g c)$ (see Fig. 1. a).

The border simplicial complex $\partial K(I)$ associated with $I$ is constructed as follows. First, we compute the $3 \mathrm{D}$ cubical complex $Q(I)$ (whose geometric building blocks are vertices, edges, squares and cubes). Second, we visit all the point of $B$, from down to up and from left to right. Let $v=(i, j, k) \in B$. If the following 7 neighbors of $v\{(i+1, j, k),(i, j+1, k),(i, j, k+1),(i+1, j+1, k),(i+1, j, k+$ $1),(i, j+1, k+1),(i+1, j+1, k+1)\}$ are also in $B$ then, the point $v$ and its 7 neighbors form a unit cube which is added to $Q(I)$ together with all its faces (vertices, edges and squares). This way, we do not consider the small artifacts of $I$. Then, the cells of the $2 \mathrm{D}$ cubical complex $\partial Q(I)$ are all the squares of $Q(I)$ which are shared by a voxel of $B$ and a voxel of $B^{c}$, together with all their faces (vertices and edges). The simplicial representation $\partial K(I)$ of $I$ is obtained from $\partial Q(I)$ by subdividing each square of $\partial Q(I)$ in 2 triangles together with all their faces (see Fig. 3,a). Finally, coordinates of the vertices of $\partial K(I)$ are normalized to coordinates $(x, y, t)$, where $0 \leq x, y \leq 1$ and $t$ is the number of silhouette of the subsequence of representation. 


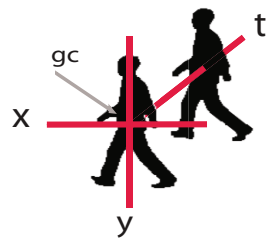

(a)

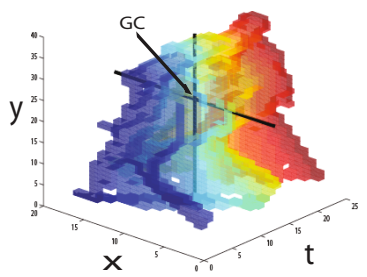

(b)

Fig. 1. (a) Silhouettes aligned by their gravity centers $(g c)$. (b) The 3D binary digital picture $I$ obtained from the silhouettes. $G C$ is the gravity center of $I$.

\section{Incremental Algorithm for Persistent Homology}

In this section, we briefly explain incremental algorithm for computing persistent homology [5. Let $K$ be a simplicial complex which is a collection of simplices (vertices, edges, triangles, tetrahedra). Incremental algorithm needs an ordering $\sigma_{1}, \sigma_{2}, \ldots, \sigma_{m}$ of the simplices of $K$. The ordering is given by a filter function that assigns a positive integer value to each simplex in $K$. The value of a simplex can not be smaller than those of its faces. Simplices of $K$ are listed in ascending order. Then, for each $i, K_{i}=\left\{\sigma_{1}, \sigma_{2}, \ldots, \sigma_{i}\right\}$ is a subcomplex of $K$. The sequence of simplices $\sigma_{i}$ (the number $i$ denotes the order of the simplex in the sequence) is called filter and the sequence of subcomplexes $\emptyset=K_{0} \subseteq K_{1} \subseteq \cdots \subseteq K_{m}=K$ filtration. When $\sigma_{i}$ is added to $K_{i-1}$, the Betti numbers of $K_{i}$ can be computed from $\sigma_{i}$ and the Betti numbers of $K_{i-1}$. If $\sigma_{i}$ completes a $d$-cycle in $K_{i}(d$ is the dimension of $\left.\sigma_{i}\right)$, then $\beta_{d}\left(K_{i}\right)=\beta_{d}\left(K_{i-1}\right)+1$. Otherwise, $\beta_{d-1}\left(K_{i}\right)=$ $\beta_{d-1}\left(K_{i-1}\right)-1$. Looking at a filtration as a growing simplicial complex, homology classes are born and die. The difference between their birth and death time is called persistence, which quantifies the significance of a topological attribute. The life interval of a homology class is a horizontal line (interval) in a plane, where birth and death time correspond to start and end points of the intervals. This representation is called persistence barcode, which is robust under noise, since features have long lives, while noise is short-lived. A Matlab implementation of the incremental algorithm of easy handling 2 or $\mathrm{C}++$ implementation 3 are available on the Internet.

\section{The New Method}

Fig. 2] shows the process chain to obtain the gait signature for gait classification. From the border simplicial complex $\partial K(I)$ associated with a gait silhouette, we obtain a concrete filter (ordering of the simplices) depending on the direction of view (see Subsection 4.1). In Subsection 4.2, the persistence barcode associated

\footnotetext{
2 http://comptop.stanford.edu/programs/plex-2.0.1-windows.zip

3 http://hg.mrzv.org/Dionysus/
} 


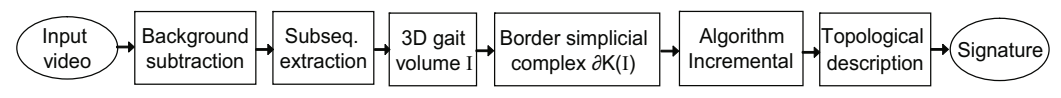

Fig. 2. Extracting the gait topological signature

with the previous filter is then computed. Only the intervals of the barcode that provide useful topological information is considered and a similarity measure for comparing two gait sequences is given.

\subsection{A Filter for $\partial K(I)$}

The topology of the border simplicial complex $\partial K(I)$ associated with a subsequence of representation is, in general, very poor. In this subsection we present a filtration on $\partial K(I)$ to get a signature using persistence barcode. This filtration captures relations among the parts of the human body when walking.

Recall that $\partial K(I)$ is a $2 \mathrm{D}$ simplicial complex, that is, only vertices, edges and triangles belong to it. Now, the triangles of $\partial K(I)$ are represented by their smallest vertices (considering the lexicographical order). If an horizontal (resp. vertical) direction is considered, then the triangles of $\partial K(I)$ are ordered by the first (resp. second) coordinate of the smallest vertex of each triangle. In general, given a direction of view $d$, a normal plane to $d$ across the origin is computed (see Fig. 3. b). Then the triangles of $\partial K(I)$ are ordered by the distance of the smallest vertex of each triangle to the plane.

Fixing a direction of view, we construct two filters $A$ and $B$. The triangles of $\partial K(I)$ (together with all their faces) are added to $A$ (resp. $B$ ) in increasing (resp. decreasing) order. Only the first half of the simplices in the filters will be considered for the topological gait signature. Let us denote these last ordered sets by $K_{[a, G C]}$ and $K_{[b, G C]}$, respectively.

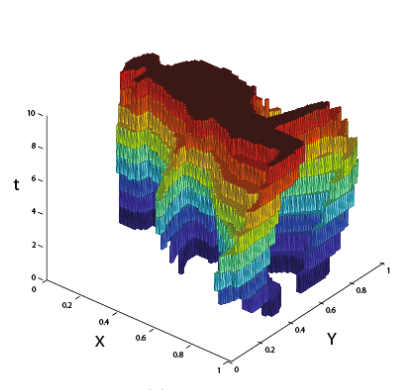

(a)

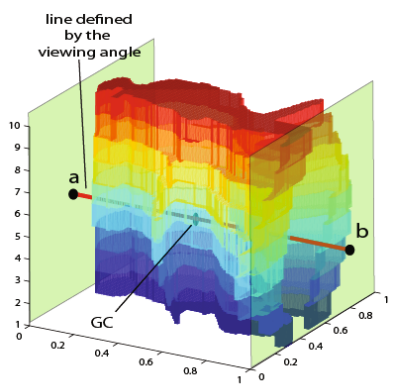

(b)

Fig. 3. (a) Border simplicial complex $\partial K(I)$. (b) A direction of view and its normal plane. 


\subsection{Topological Gait Signature}

The gait signature is obtained based on the topological invariants extracted from persistence barcode after removing the intervals that do not provide useful information.

First, persistence barcodes are computed for $K_{[a, G C]}$ and $K_{[b, G C]}$. Second, we reduce the barcodes removing the intervals $[i, j]$ such that the number of triangles in $[i, j-1]$ is less than 2 (i.e., homology classes with low persistence are ignored).

Now, $n$ cuts are performed homogeneously in the sets $K_{[a, G C]}$ and $K_{[b, G C]}$, being $n$ a given positive integer, as follows: Suppose $K_{[a, G C]}=\left\{\sigma_{0}, \ldots, \sigma_{m}\right\}$. Construct the subsets $P_{i}^{a}=\left\{\sigma_{\left\lfloor\frac{(i-1) m}{n}\right\rfloor+1}, \ldots, \sigma_{\left\lfloor\frac{i m}{n}\right\rfloor-1}\right\}, 1 \leq i \leq n$. Fixed $i$, the reduced persistence barcode shows: (a) Homology classes that were born or persist when the simplex $\sigma_{\left\lfloor\frac{(i-1) m}{n}\right\rfloor}$ is added, and, persist or die when the simplex $\sigma_{\left\lfloor\frac{i m}{n}\right\rfloor}$ is added. (b) Homology classes that were born in $P_{i}^{a}$. An analogous process is done for $K_{[b, G C]}$. A vector of dim. $2 n$ is then formed counting the number of homology classes classified as explained above.

For example, consider the border simplicial complex $\partial K(I)$ given in Fig. 4 which consists in 136474 simplices. We perform $n=5$ cuts on the set $K_{[a, G C]}$ (resp. $\left.K_{[b, G C]}\right)$. See the green lines in the persistence barcode representations in Fig. 4 . For instance, consider the set $K_{[b, G C]}$ and fix $i=2$. According to Fig. 4 , the number of homology classes that persist or were born in $\sigma_{13648}$, and, persist or die in $\sigma_{27296}$ are $H_{0}=5 \mathrm{in} \operatorname{dim} .0$ and $H_{1}=1 \mathrm{in} \operatorname{dim}$. 1 . The number of the homology classes that were born in $P_{2}^{b}$ are $H_{0}=2$ in dim. 0 and $H_{1}=5$ in dim. 1 .

The topological signature for a gait subsequence considering a fixed direction of view consists in four $2 n$-dimensional vectors: $\left(V_{1}, V_{2}, V_{3}, V_{4}\right)$ constructed as explained above.

In our example, we have four 10-dimensional vectors: $V_{1}$ (resp. $V_{2}$ ) is the first (resp. second) column of the table on the left in Fig. 4. $V_{3}$ (resp. $V_{4}$ ) is the first (resp. second) column of the table on the right in Fig. 4. The similarity value for the topological signatures $\left(V_{1}, V_{2}, V_{3}, V_{4}\right)$ and $\left(W_{1}, W_{2}, W_{3}, W_{4}\right)$ for two gait subsequences considering a fixed direction of view is done by computing, first, the angle between the two vectors $V_{i}$ and $W_{i}$, for $i=1,2,3,4$ using Eq. 1.

$$
S_{i}=\cos ^{-1}\left(\frac{V_{i} \cdot W_{i}}{\left\|V_{i}\right\| \quad\left\|W_{i}\right\|}\right)
$$

Then, four angles $\left(S_{1}, S_{2}, S_{3}, S_{4}\right)$ are obtained, since for each subsequence of representation, four vectors were computed. The total similarity value for two gait subsequences considering a fixed direction of view, $\mathrm{O}_{1}$ and $\mathrm{O}_{2}$, is the weighted sum of the four similarity measures (angles) computed before:

$$
S\left(O_{1}, O_{2}\right)=w_{1} S_{1}+w_{2} S_{2}+w_{3} S_{3}+w_{4} S_{4}
$$




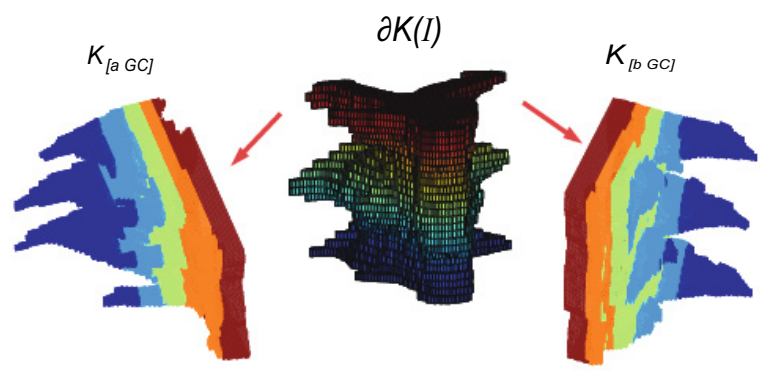

\begin{tabular}{|c|c|c|}
\hline \multicolumn{3}{|c|}{$\boldsymbol{K} \boldsymbol{a} \boldsymbol{G} \boldsymbol{C}]$} \\
\hline$P$ & $H_{O}$ & $H_{I}$ \\
\hline \multirow{2}{*}{$P_{1}$} & 1 & 0 \\
\cline { 2 - 3 } & 7 & 13 \\
\hline \multirow{2}{*}{$P_{2}$} & 6 & 11 \\
\cline { 2 - 3 } & 2 & 5 \\
\hline \multirow{2}{*}{$P_{3}$} & 3 & 16 \\
\cline { 2 - 3 } & 3 & 6 \\
\hline \multirow{2}{*}{$P_{4}$} & 3 & 19 \\
\cline { 2 - 3 } & 1 & 6 \\
\hline \multirow{2}{*}{$P_{5}$} & 3 & 20 \\
\cline { 2 - 3 } & 1 & 1 \\
\hline
\end{tabular}
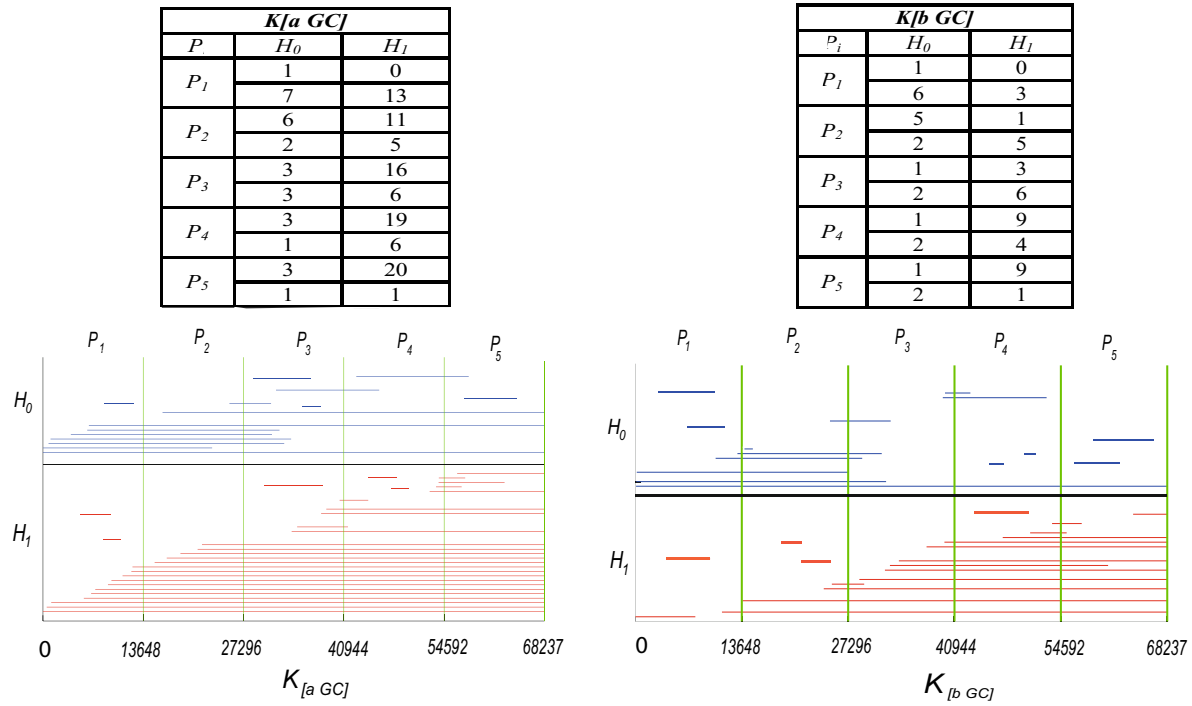

Fig. 4. Reduced persistence barcodes filtered according to the direction of view given in Fig. 3.b. Five cuts are done on the sets $K_{[a, G C]}$ and $K_{[b, G C]}$.

\section{$5 \quad$ Experimental Results}

In this section, we test the proposed method on 11 directions of view of the CASIA-B database, which contains 124 subjects. There are 6 normal walking sequences for each person. CASIA-B database provides image sequences with background subtraction for each person.

In this experiment the subsequence of representation is all the walking sequence, which approximately consists in two gait cycles. We have used 4 direction of view: The first one is horizontal (axe $X$ ), the second one is vertical (axe $Y)$, the third one forms 45 degrees with the axes $X$ and $Y$ and is orthogonal to the axe $T$ (see Section 2). The fourth direction of view is orthogonal to the axe $T$ and to the third direction of view. See Fig. 5 . Besides, in our experiment, the number of cuts in each direction of view is $n=23$ (see Subsection 4.2). Finally, the value of $w_{i}, i=1,2,3,4$, according to Eq. 2 is 1 . 


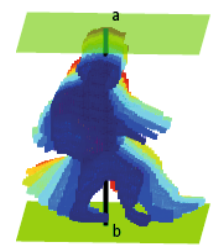

(a)

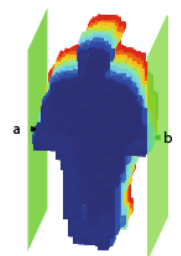

(b)

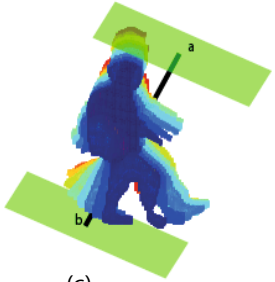

(c)

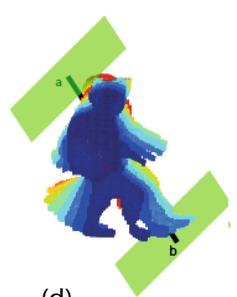

(d)

Fig. 5. Directions of view used in our experiments

The experiment was carried out using 4 sequences for training, and 2 as testing set. The results are compared with the ones in 6 6 1310. Table. 1 shows the cross validation average (15 combinations) of correct classification rates at rank 1 from the candidate list. It is also considered the correct classification rate when at least one subject of the two used as test is correctly classified (ALS). The correct classification rate (CCR) provides accuracy over the whole test set. However, the at-least-one subject criterion (ALS) shows an idea of the accuracy by classes.

Table 1. Correct classification rates (CCR in \%)

\begin{tabular}{lllllllllllll}
\hline Method & 0 & 18 & 36 & 54 & 72 & 90 & 108 & 126 & 144 & 162 & 180 & Avg \\
\hline Goffredo. M, et al [6] & - & - & 72.1 & 79.5 & 85.0 & 86.5 & 82.3 & 81.1 & - & - & - & 81.1 \\
Wavelet(FD) [3] & 100 & 100 & 100 & 93.4 & 81.1 & 90.3 & 90.3 & 83.3 & 91.9 & 92.7 & 97.6 & 93.0 \\
Gait energy image [10] & 99.2 & 99.6 & 97.6 & 97.2 & 97.2 & 97.6 & 95.6 & 96.8 & 96.4 & 98.4 & 99.6 & 97.7 \\
Our method & & & & & & & & & & & & \\
rank 1 & 99.3 & 99.1 & 98.8 & 98.3 & 97.6 & 98.0 & 98.3 & 98.3 & 98.2 & 98.2 & 99.0 & 98.5 \\
ALS 1 & 100 & 100 & 100 & 100 & 99.0 & 100 & 100 & 100 & 99.4 & 98.9 & 99.1 & 99.7 \\
\hline
\end{tabular}

To evaluate the discriminative power of the features, we use the first 50 persons from 90 degree view of the CASIA-B data base. The evaluation was carried out

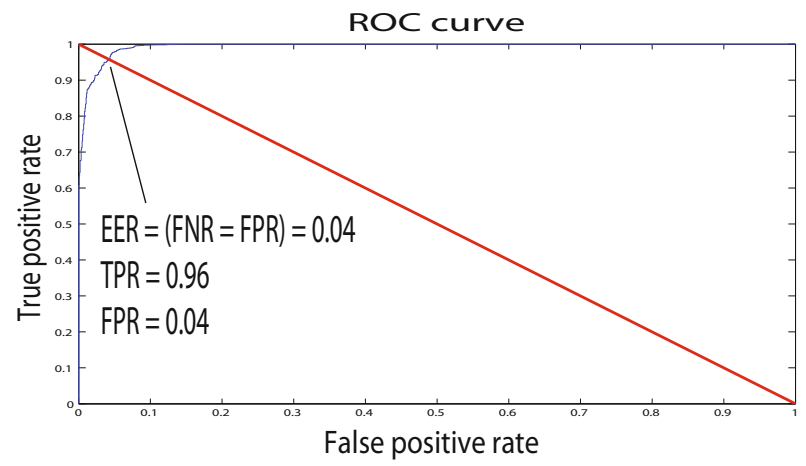

Fig. 6. Receiver operating characteristic (ROC) curve 
using 4 sequences for training, and 2 as testing set. Fig. 6 shows the receiver operating characteristic (ROC) curve, which provides detailed information on the trade-off between the two types of errors, False Positive Rate (FPR) and False Negative Rate (FNR). The intersection of the ROC curve with the diagonal (see Fig. 6, blue line) is the means of the equal error rate (EER), i.e, where $F P R=F N R$. In our experiment $\mathrm{EER}=0.04, \mathrm{FPR}=0.04$ and therefore, True Positive Rate (TPR) is 0.96 .

\section{Conclusion and Future Work}

In this paper we propose a new representation based on topological invariants for human gait recognition. A new approach called reduced persistence barcode is used to improve the discriminative capacity of the representation. This approach adds the possibility to use more discriminative topological invariants such as cup product [7. Furthermore, we plan to use the bottleneck distance as similarities measures between barcodes, which guarantees stability in Persistent Homology. For this, we need a filtration associated to a continuous tame real-valued function [4. We want to extend these experiments using other database. On the other hand, we want to evaluate the diversity in order to combine classifiers based on geometric and topological features.

\section{References}

1. Bouchrika, I., Goffredo, M., Carter, J.N., Nixon, M.S.: Covariate Analysis for ViewPoint Independent Gait Recognition. In: Tistarelli, M., Nixon, M.S. (eds.) ICB 2009. LNCS, vol. 5558, pp. 990-999. Springer, Heidelberg (2009)

2. Carlsson, G., Zomorodian, A., Collins, A., Guibas, L.: Persistence barcodes for shapes. In: Proc. of SGP 2004, pp. 124-135 (2004)

3. Chen, C.H., Liang, J.M., Zhao, H., Hu, H.H., Tian, J.: Frame difference energy image for gait recognition with incomplete silhouettes. PRL 30(11) (2009)

4. Cohen-Steiner, D., Edelsbrunner, H., Harer, J.: Stability of persistence diagrams. Discrete \& Computational Geometry 37(1) (2007)

5. Edelsbrunner, H., Letscher, D., Zomorodian, A.: Topological persistence and simplification. Discrete \& Comput. Geometry 28(4), 511-533 (2002)

6. Goffredo, M., Bouchrika, I., Carter, J.N., Nixon, M.S.: Self-calibrating viewinvariant gait biometrics. IEEE Transactions on Systems, Man, and Cybernetics, Part B 40(4), 997-1008 (2010)

7. Gonzalez-Diaz, R., Umble, R., Lamar-León, J.: Cup Products on Polyhedral Approximations of 3D Digital Images. In: Aggarwal, J.K., Barneva, R.P., Brimkov, V.E., Koroutchev, K.N., Korutcheva, E.R. (eds.) IWCIA 2011. LNCS, vol. 6636, pp. 107-119. Springer, Heidelberg (2011)

8. Hilaga, M., Shinagawa, Y., Kohmura, T., Kunii, T.L.: Topology matching for fully automatic similarity estimation of $3 \mathrm{~d}$ shapes. In: Proc. of the $28 \mathrm{th}$ Conf. on Computer Graphics and Interactive Techniques, SIGGRAPH 2001, pp. 203-212 (2001)

9. Nixon, M.S., Carter, J.N.: Automatic recognition by gait. Proc. of IEEE 94(11), 2013-2024 (2006)

10. Yu, S.Q., Tan, D.L., Tan, T.N.: A framework for evaluating the effect of view angle, clothing and carrying condition on gait recognition. In: ICPR, pp. IV:441-IV:444 (2006) 